\title{
Improved lung allograft function after fundoplication in patients with gastroesophageal reflux disease undergoing lung transplantation
}

\author{
R. Duane Davis, Jr, MD \\ Christine L. Lau, MD ${ }^{a}$ \\ Steve Eubanks, MD \\ Robert H. Messier, MD, PhDa \\ Denis Hadjiliadis, MD $^{\mathrm{b}}$ \\ Mark P. Steele, MD \\ Scott M. Palmer, MD
}

From the Departments of Surgery a and Pulmonary Medicine, ${ }^{\mathrm{b}}$ Duke University Medical Center, Durham, NC.

Read at the Eighty-second Annual Meeting of The American Association for Thoracic Surgery, Washington, DC, May 6-8, 2002.

Received for publication May 10, 2002; revisions requested July 17, 2002; revisions received Aug 29, 2002; accepted for publication Sept 12, 2002.

Address for reprints: R. Duane Davis, MD, Department of Thoracic Surgery, Duke University Medical Center, Box 3864, Durham, NC $27710 \quad$ (E-mail: davis053@mc.duke.edu).

J Thorac Cardiovasc Surg 2003;125:533-42

Copyright (C) 2003 by The American Association for Thoracic Surgery

$0022-5223 / 2003 \$ 30.00+0$

doi: $10.1067 / \mathrm{mtc} .2003 .166$
Objectives: Bronchiolitis obliterans is the greatest limitation to the long-term applicability of lung transplantation. Although alloimmune events are important, nonimmune events, such as gastroesophageal reflux, might contribute to lung injury and the development of bronchiolitis obliterans syndrome.

Methods: We retrospectively studied the 396 patients who underwent lung transplantation at the Duke Lung Transplant Program from April 1992 to April 2002. Reflux was assessed for using an ambulatory 24-hour esophageal $\mathrm{pH}$ probe.

Results: Reflux assessment with an esophageal pH probe was obtained in 128 patients after lung transplantation. Abnormal pH study results were present in 93 (73\%) patients. Forty-three patients underwent a surgical fundoplication. There was no in-hospital or 30-day mortality in the patients undergoing fundoplication. At the time of fundoplication, 26 patients met the criteria for bronchiolitis obliterans syndrome. After fundoplication, 16 patients had improved bronchiolitis obliterans syndrome scores, with 13 of these patients no longer meeting the criteria for bronchiolitis obliterans syndrome. In patients at least 6 months after lung transplantation and 6 months after fundoplication, the forced expiratory volume in 1 second improved by an average of $24 \%$ (mean forced expiratory volume in 1 second before fundoplication, $1.87 \mathrm{~L}$; mean forced expiratory volume in 1 second after fundoplication, $2.19 \mathrm{~L} / \mathrm{sec} ; P<.0002$ ). Overall actuarial survival was significantly better in patients who had either normal $\mathrm{pH}$ studies or who had fundoplication.

Conclusions: Gastroesophageal reflux disease is very common after lung transplantation and appears to contribute to mortality and development of bronchiolitis obliterans syndrome. Fundoplication in lung transplant recipients with gastroesophageal reflux disease is associated with significant improvements in lung function, particularly if performed before the late stages of bronchiolitis obliterans syndrome.

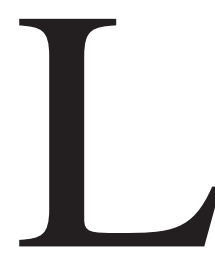

ung transplantation has evolved into an effective treatment for patients with end-stage lung disease, with many centers reporting greater than $80 \%$ 1-year survival. As immediate posttransplant surgical outcomes have improved, the greatest limitation of lung transplantation remains chronic allograft dysfunction or bronchiolitis obliterans syndrome (BOS). Five-year patient survival remains a disappointing $40 \% .^{1,2}$

BOS is a clinical syndrome manifested by decreases in the forced expiratory volume in 1 second $\left(\mathrm{FEV}_{1}\right)^{3,4} \mathrm{BOS}$ is a surrogate marker for chronic rejection, 
which is manifested pathologically by scar formation and fibrosis of the small airways that defines the pathologic entity termed obliterative bronchiolitis. The incidence of BOS increases with time and is greater than $60 \%$ by 5 years after transplantation. ${ }^{5-8}$ The lung, as compared with other organs, appears to be significantly more susceptible to chronic injury. Although 5-year allograft survival from the United Network for Organ Sharing (UNOS) registry is 68\% and $63 \%$ for the heart and cadaveric kidney, respectively, lung allograft survival was only $42 \%{ }^{1}$

The pathogenesis of chronic lung dysfunction is poorly understood. Although chronic immunologic injury is implicated, treatment of patients with bronchiolitis obliteransBOS by augmenting or altering immunosuppression rarely reverses the lung dysfunction. Several therapies have been attempted, and although a few have resulted in stabilization of the patients' pulmonary status, results from treatment have been generally disappointing. Despite attempts to reverse or stabilize the patient's pulmonary status, progression of the chronic rejection process occurs frequently.9-18

Previous studies have identified several risk factors for the development of BOS, including frequent, severe, or both early acute rejection episodes; cytomegalovirus (CMV) infection and pneumonia; mismatches at human HLA loci; and development of antibodies to class I HLA..$^{719-22}$ Recent reports have suggested that gastroesophageal reflux with resultant aspiration could contribute to the allograft dysfunction, representing a potential cause of BOS. ${ }^{23,24}$

Gastroesophageal reflux disease (GERD) has a high prevalence in patients with a variety of lung diseases, particularly patients with asthma, cystic fibrosis, and pulmonary fibrosis. ${ }^{25-30}$ In patients after pneumonectomy, there are marked abnormalities in esophageal function and reflux. ${ }^{31}$ GERD has been associated with the development of bronchiolitis obliterans with organizing pneumonia. ${ }^{32}$ For multiple reasons (the use of immunosuppressive drugs and vagal nerve injury during the operation), GERD might be increased after lung transplantation. Additionally, host defense mechanisms of the lung, including the cough reflux and mucociliary clearance of foreign bodies, is markedly impaired. ${ }^{33-37}$

In this study we hypothesize that GERD causing repetitive gastric aspiration produces chronic lung injury and that prevention of gastric reflux with fundoplication would improve lung function.

\section{Patients and Methods}

We retrospectively analyzed 396 consecutive lung transplantations performed at Duke University Medical Center from April 1992 to April 2002. Standard operative techniques were used. Demographic data, including time of transplant, sex, age, type, and survival, was collected on all patients. For comparison, allograft survival data for kidney and heart transplants performed at Duke from 1990 to 2002 were obtained. Lung transplant recipients underwent routine $\mathrm{FEV}_{1}$ measurements at each clinic visit or at any time if they showed clinical evidence of deterioration. The most recent $\mathrm{FEV}_{1}$ values were then compared with the best $\mathrm{FEV}_{1}$ value obtained by the patients after transplantation.

The diagnosis of chronic rejection, BOS, was made on the basis of pulmonary function data according to International Society for Heart and Lung Transplantation (ISHLT) criteria. ${ }^{3}$ The diagnosis of BOS requires a $20 \%$ or greater decrease in pulmonary function from a patient's baseline value and the exclusion of other causes of decreasing lung function, including narrowing of the anastomosis, acute rejection, or infection. Recipients were considered at risk for BOS only if they survived 6 or more months after transplantation. For the analysis, a BOS score of 1 or greater was considered positive, whereas a BOS score of 0 was considered negative.

A subset of this series of lung transplantation had been evaluated for GERD, and data on this group, including date of $\mathrm{pH}$ study and result, were included. A subset of this smaller group underwent treatment for GERD by means of surgical fundoplication, and data, including results, prefundoplication and postfundoplication $\mathrm{FEV}_{1}$ values, and survival, were tabulated on this series. Standard laparoscopic techniques were used for the majority of fundoplications, and when converted to open procedures, standard operative techniques were used.

\section{pH Probe Studies}

GERD was assessed in 128 patients after lung transplantation by using 24-hour ambulatory $\mathrm{pH}$ probe studies. Proton pump inhibitors were discontinued for at least 5 days before the $\mathrm{pH}$ studies. Histamine (H-2) blockers and promotility agents were stopped at least 24 hours before the studies. The esophageal $\mathrm{pH}$ laboratory at Duke University Medical Center uses standard techniques. ${ }^{38} \mathrm{~A}$ distal esophageal probe was inserted in a standardized manner by one technician in all patients. The probe was removed after approximately 24 hours. Patients were instructed to go on with their daily activities as usual. A small number of nonselected patients also had a proximal $\mathrm{pH}$ probe placed in the esophagus. The correlation between the distal and the proximal probe measurements was good, and therefore there was no need for a proximal probe in all patients. Abnormal acidity in the esophagus was recorded every time the $\mathrm{pH}$ decreased to less than 4 . The results were reported as the percentage of abnormal acid contact time at the distal esophagus. Normal values for acid contact times were as follows: total less than $5 \%$, upright less than $8 \%$, and supine less than $3 \%$.

Early in the series, patients selected for $\mathrm{pH}$ studies were suspected of having GERD on the basis of symptoms, clinical concerns (eg, vocal cord erythema and inflammation on bronchoscopy or foreign material consistent with aspiration on biopsy), or an unexplained decrease in $\mathrm{FEV}_{1}$ value. From March 1998 until July 2000, patients were studied prospectively under an institutional review board protocol. Because of the high prevalence of GERD and demonstrable improvements in $\mathrm{FEV}_{1}$ values in patients with GERD after fundoplication, from July 2000, pH studies were performed liberally as part of routine clinical practice.

\section{Immunosuppression}

After lung transplantation, all patients received similar immunosuppression. From 1992 until May 2000, patients received cyclo- 
TABLE 1. Lung transplant patients at Duke

\begin{tabular}{|c|c|c|c|}
\hline & $\begin{array}{l}\text { Lung transplant } \\
\text { with reflux }\end{array}$ & $\begin{array}{l}\text { Lung transplant } \\
\text { without reflux }\end{array}$ & $P$ value \\
\hline No. of patients & $93(73 \%)$ & $35(27 \%)$ & \\
\hline Average age (y) & $47 \pm 14$ & $51 \pm 12$ & NS \\
\hline \multicolumn{4}{|l|}{$\operatorname{Sex}(\%)$} \\
\hline Female & $41(44)$ & $21(60)$ & NS \\
\hline Male & $52(5)$ & $14(40)$ & NS \\
\hline \multicolumn{4}{|l|}{ Underlying disease (\%) } \\
\hline CF/bronchiectasis & $22(24)$ & $7(20)$ & NS \\
\hline COPD/A1A def & $51(55)$ & $21(60)$ & NS \\
\hline OB/Rtx & $2(2)$ & $1(3)$ & NS \\
\hline IPF & $9(10)$ & $2(6)$ & NS \\
\hline PPH & $2(2)$ & $1(3)$ & NS \\
\hline Other & $7(8)$ & $3(9)$ & NS \\
\hline \multicolumn{4}{|l|}{ Type of transplant (\%) } \\
\hline Bilateral & $57(61)$ & $26(74)$ & NS \\
\hline Single & $36(39)$ & $9(26)$ & NS \\
\hline
\end{tabular}

$C F$, Cystic fibrosis; $C O P D$, chronic obstructive pulmonary disease; $A I A$ def, $\alpha_{\mathrm{i}}$-antitrypsin deficiency; $0 B$, obliterative bronchiolitis; $R t x$, retransplantation; IPF, idiopathic pulmonary fibrosis; $P P H$, primary pulmonary hypertension; $N S$, not significant.

sporine (INN: ciclosporin; 5-10 $\mathrm{mg} \times \mathrm{kg}^{-1} \times \mathrm{d}^{-1}$ ), aiming for a target trough level of 250 to $300 \mathrm{ng} / \mathrm{mL}$ (high-performance liquid chromatography). After May 2000, tacrolimus was used initially sublingually followed by oral administration, aiming for a target trough level between 10 and $15 \mathrm{ng} / \mathrm{mL}$. Azathioprine (1-2 mg $\times$ $\mathrm{kg}^{-1} \times \mathrm{d}^{-1}$ ) was used throughout. From 1997 to 1998 , patients received either azathioprine or mycophenolate ( $1 \mathrm{~g}$ twice daily) as part of an ongoing randomized study in addition to cyclosporine or prednisone. Patients received intravenous methylprednisolone (500 mg per lung intraoperatively, followed by $125 \mathrm{mg}$ every 12 hours for 4 doses), followed by prednisone (initially at $20 \mathrm{mg} / \mathrm{d}$ ). Induction therapy with rabbit antithymocyte globulin was used in 22 patients from 1994 to 1996 as part of a previous randomized study. From January 1999, induction therapy with an anti-CD25 monoclonal antibody $(1 \mathrm{mg} / \mathrm{kg}$ daclizumab or $20 \mathrm{mg}$ of basiliximab on days 0 and 4) was used.

Episodes of acute allograft rejection were treated with 500 $\mathrm{mg} / \mathrm{d}$ methylprednisolone for 3 days, followed by a 2-week oral prednisone taper. Refractory or persistent rejection was treated with rabbit antithymocyte globulin, antithymocyte gamma-globulin, or, most recently, alemtuzumab. Additionally, cyclosporine was changed to tacrolimus and azathioprine to mycophenolate mofetil in many patients.

\section{Infection Prophylaxis}

All patients at risk for CMV infection (positive donor or recipient serology) received prophylaxis with ganciclovir. All patients received Pneumocystis carinii prophylaxis indefinitely. Fungal infection prophylaxis consisted of nystatin swish and swallow for the first 6 months after transplantation. In addition, since January 1997, aerosolized amphotericin B (either liposomal or conventional) was given for at least 2 weeks after transplantation. Vancomycin and ceftazidime were used for bacterial infection prophylaxis the first 2 weeks after transplantation. In patients with septic lung disease, antibiotic choice was individualized and guided by pretransplant cultures.

\section{Statistical Analysis}

Differences between $\mathrm{FEV}_{1}$ values before and after fundoplication were compared by using a paired 2-tailed $t$ test. Demographic data between groups was assessed by using $\chi^{2}$ analysis. Overall survival was determined by using Kaplan-Meier actuarial analysis. Differences in actuarial freedom between groups were determined with the log-rank test. Data analysis was performed with NCSS 2000 software (NCSS, Kaysville, Utah). Values were reported as means \pm SEM except for age, which was reported as means \pm SD.

\section{Results}

In the decade from its inception in April 1992, The Duke Lung Transplant Program has performed 396 lung transplantations. Since 1997, we have performed $\mathrm{pH}$ studies on 128 patients after lung transplantation. Forty-three patients have subsequently undergone fundoplication surgery.

\section{pH Studies}

Of the 128 patients evaluated with a 24-hour ambulatory $\mathrm{pH}$ probe, $93(73 \%)$ had abnormal $\mathrm{pH}$ study results. In patients with abnormal $\mathrm{pH}$ study results, the average total, supine, and upright values were $13.1 \% \pm 0.9 \%, 14.3 \% \pm 1.1 \%$, and $11.9 \% \pm 1.2 \%$, respectively. For the $35(27 \%)$ patients with normal $\mathrm{pH}$ studies, the average total, supine, and upright values were $2.0 \% \pm 0.3 \%, 2.8 \% \pm 0.4 \%$, and $0.5 \% \pm$ $0.2 \%$, respectively. There was no statistical difference in respect to age, sex, transplant type, or pretransplant diagnosis between the patients with normal and those with abnormal $\mathrm{pH}$ study results (Table 1).

The $\mathrm{pH}$ study results were obtained an average of 507 days (range, 29-2502 days) after transplantation. At the time of the studies, $33(26 \%)$ of the patients met the criteria for BOS. 
TABLE 2. Lung transplant patients at Duke

\begin{tabular}{|c|c|c|c|}
\hline & $\begin{array}{l}\text { Antireflux } \\
\text { surgery }\end{array}$ & $\begin{array}{c}\text { No antireflux } \\
\text { surgery }\end{array}$ & $P$ value \\
\hline No. of patients & 43 & 353 & \\
\hline Average age (y) & $43.7 \pm 15(16-66)$ & $47.7 \pm 14(10-66)$ & NS \\
\hline \multicolumn{4}{|l|}{ Sex $(\%)$} \\
\hline Female & $18(42)$ & $173(49)$ & NS \\
\hline Male & $25(58)$ & $180(51)$ & NS \\
\hline \multicolumn{4}{|c|}{ Underlying disease (\%) } \\
\hline Cystic fibrosis & $13(30)$ & $65(18)$ & NS \\
\hline Bronchiectasis & $2(5)$ & $5(1)$ & NS \\
\hline $\mathrm{OB} / \mathrm{Rtx}$ & $3(7)$ & $9(3)$ & NS \\
\hline COPD/A1Adef & $15(35)$ & $172(49)$ & NS \\
\hline IPF & $5(12)$ & $46(13)$ & NS \\
\hline $\mathrm{PPH}$ & $1(2)$ & $17(5)$ & NS \\
\hline Other & $4(9)$ & $39(11)$ & NS \\
\hline \multicolumn{4}{|c|}{ Type of transplant (\%) } \\
\hline Bilateral & $30(70)$ & $216(61)$ & NS \\
\hline Single & $13(30)$ & $137(39)$ & NS \\
\hline
\end{tabular}

For legend see Table 1.

Of the patients studied who met the criteria for BOS at the time of their $\mathrm{pH}$ study, $25(76 \%)$ had abnormal $\mathrm{pH}$ values, and $8(24 \%)$ had normal $\mathrm{pH}$ values.

\section{Fundoplication Group}

We have performed antireflux operations in 43 patients undergoing lung transplantation, with the majority of operations being laparoscopic Nissens. Three cases were performed as open procedures because of extensive adhesions, and 4 laparoscopic toupee wraps were performed. In 10 patients abnormal gastric emptying was demonstrated in an abnormal nuclear medicine solid gastric emptying study. In these patients the following additional gastric drainage procedures were performed: there were 6 pyloroplasties and 1 gastrojejunostomy, and 2 patients received both a gastrostomy tube for drainage and a jejunostomy tube for alimentary access. One patient did not receive any additional drainage procedure. There was no in-hospital or 30-day mortality after the fundoplication operations.

Indications for fundoplication were an abnormal $\mathrm{pH}$ study in 39 patients, severe reflux documented by barium swallow but with normal esophageal manometry in 2 patients, retransplant for a patient with presumed repetitive aspiration based on pathology of the explanted lung demonstrating predominantly lipid pneumonia, and in 1 patient on the basis of repetitive episodes of aspiration pneumonia. In the patients with $\mathrm{pH}$ studies, the percentages of average acid contact times were as follows: $17.5 \% \pm 1.2 \%, 18.7 \%$ $\pm 1.6 \%$, and $15.5 \% \pm 2.0 \%$ for the total, upright, and supine values, respectively.

In terms of demographics between the lung transplant recipients undergoing a fundoplication operation and the overall series, there were no significant differences between the 2 groups in terms of age, sex, type of transplant, or pretransplant diagnosis (Table 2).

In terms of risk factors for chronic allograft rejection, there was no difference between the 2 groups in terms of increased pretransplant panel-reactive antibody screening, CMV infections, or mismatches at HLA loci. There were more episodes of early and severe acute rejection episodes seen in the fundoplication group $(P=.04)$.

\section{$\mathrm{FEV}_{\mathbf{1}} / \mathrm{BOS}$}

In the lung transplant recipients who underwent fundoplication, the $\mathrm{FEV}_{1}$ value significantly improved after the surgical treatment for documented GERD. The average $\mathrm{FEV}_{1}$ value before antireflux surgery was $1.87 \mathrm{~L}$, and when re-evaluated at least 6 months after antireflux surgery, the $\mathrm{FEV}_{1}$ value had increased to $2.19 \mathrm{~L}$, representing an increase of $24.1 \%$ (Figure 1). Greater than $80 \%$ of lung transplant recipients who also underwent antireflux surgery for documented GERD had improvements in their $\mathrm{FEV}_{1}$ values after fundoplication.

Of the 43 patients undergoing fundoplication, 26 (60\%) met he criteria for the development of BOS at the time of their antireflux operation. Of these, $13(50 \%)$ patients met the criteria for BOS 1, $7(27 \%)$ for BOS 2, and $6(23 \%)$ for BOS 3. Seventeen (40\%) patients did not meet the criteria for BOS. All patients free from BOS at the time of fundoplication remained so after fundoplication. Ten $(77 \%)$ of the 13 patients with BOS improved their $\mathrm{FEV}_{1}$ values enough that they no longer met the criteria for BOS after fundoplication. Forty-three percent of patients with BOS 2 at the time of fundoplication had sufficient improvement of their $\mathrm{FEV}_{1}$ values that they no longer met the criteria for BOS after fundoplication, whereas another 28\% improved to 


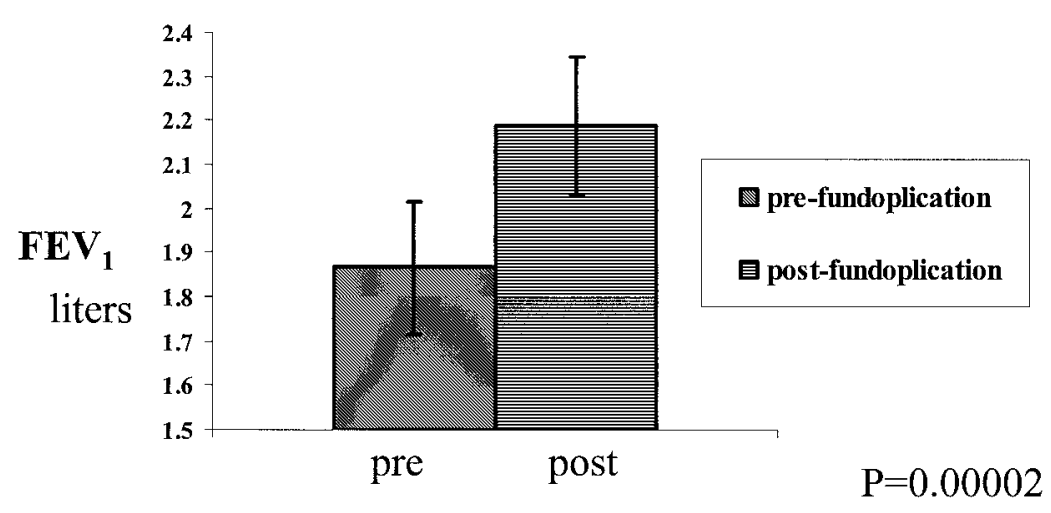

Figure 1. Average FEV 1 values in lung transplant recipients who also underwent fundoplication surgery for treatment of reflux. Patients were at least 6 months from both their lung transplantations and fundoplication procedures to allow stabilization of pulmonary function. A significant improvement in $F E V_{1}$ was documented in this group after fundoplication.

BOS 1. Only $17 \%$ of patients with BOS 3 at the time of fundoplication had improvement in their BOS score.

\section{Survival}

Normal versus abnormal pH studies. Survival, as determined by means of Kaplan-Meier analysis, in the patients with normal $\mathrm{pH}$ study results was significantly improved compared with that of patients with abnormal $\mathrm{pH}$ study results $(P=.047$, Figure 2). Overall survival was similar at 1 year (94\% in the normal $\mathrm{pH}$ group and $96 \%$ in the reflux group); however, survival was substantially better at 3 and 5 years after transplantation in patients with normal $\mathrm{pH}$ study results ( $91 \%$ and $82 \%$, respectively) compared with in those with reflux (77\% and $48 \%$, respectively).

Fundoplication group. Survival, as determined by means of Kaplan-Meier analysis, in the fundoplication group was significantly improved compared with that in the routine transplant population $(P=.013)$. Overall 1-year survival in the fundoplication group was $95 \%$ versus $78 \%$ in the overall series. Three- and 5-year survival was $86 \%$ and $71 \%$ in the fundoplication group compared with $69 \%$ and $48 \%$ in the overall series (Figure 3).

Kidney, heart, and lung allograft survival. KaplanMeier actuarial survival was performed on 6-month survivors of kidney, heart, and lung allografts performed after 1990 at Duke to correct for early differences in outcomes between heart, kidney, and lung transplants as a result of technical reasons. A significantly worse outcome for patients undergoing lung transplantation occurred (Figure 4, A). In contradistinction, patients undergoing lung transplantation documented to be without reflux or whose reflux was treated with fundoplication have allograft survival equivalent to that of patients who have undergone a kidney or heart transplantation (Figure 4, B).

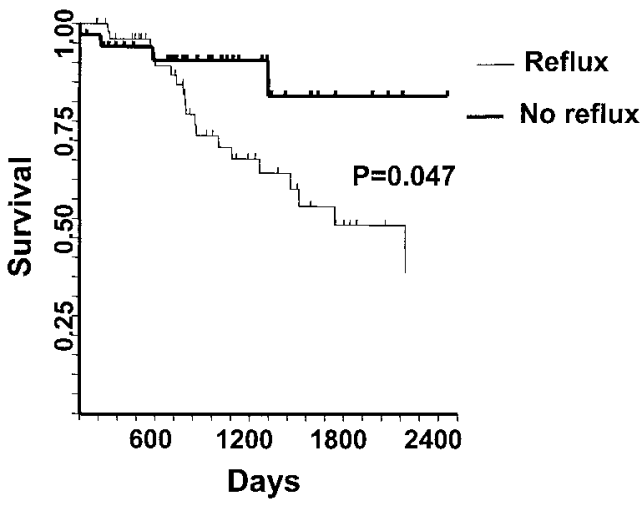

Figure 2. Effect of GERD on survival: Kaplan-Meier actuarial survival curves for overall lung transplant recipients compared with the group of lung transplant recipients who also underwent fundoplication surgery after being evaluated for GERD. The fundoplication group had a significant survival advantage.

\section{Discussion}

There is a high incidence and prevalence of GERD in patients with end-stage lung disease awaiting lung transplantation. This is particularly true for those patients with pulmonary fibrosis, in whom the incidence is greater than 50\%. ${ }^{25,39}$ However, after lung transplantation, the incidence increases to three fourths of the patients. The increase in reflux presumably is multifactorial, including vagus nerve injury or dysfunction, effects of immunosuppression medication on lower esophageal sphincter function, and relative changes in intrathoracic and intra-abdominal pressures.

The consequence of GERD in patients undergoing lung transplantation with denervated lungs, markedly impaired cough reflex, and abnormal mucociliary function is that 


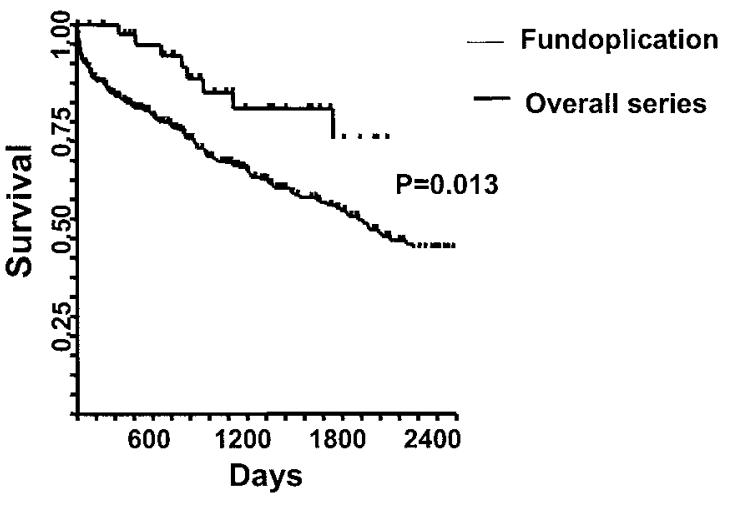

Figure 3. Effect of fundoplication on survival. Kaplan-Meier actuarial survival curves for patients evaluated for reflux by 24-hour pH studies, comparing the group with documented reflux versus the group with no reflux. A significant survival advantage was seen in the patients with normal $\mathrm{pH}$ studies.

prolonged contact time of aspirated gastric contents might lead to substantially greater lung parenchymal injury. Although GERD might cause repetitive direct lung injury, it is also possible that it plays a role in augmenting the alloimmune response. This could occur through a number of mechanisms. Injury to the airway epithelium causes activation and upregulation of major histocompatibility complex class II expression. This could lead to a greater amount of antigen presentation, either through direct or indirect antigen-presentation pathways. Cell damage would increase the amount of peptide available for presentation through indirect antigen-presentation pathways. Additionally, the normal inflammatory response to aspiration injury would bring a number of inflammatory cells, including macrophages and monocytes, which, in addition to their ability to function as professional antigen-presenting cells, can also provide the appropriate costimulatory and proliferative signals to greatly augment an alloimmune response.

The prevention of GERD by using fundoplication might have a significant effect on both direct parenchymal injury and on decreasing the amount of alloimmune injury. From our data, it appears that fundoplication was particularly successful in improving allograft function in patients who had the earliest stages of BOS. It is likely that by eliminating airway injury caused by aspiration of gastric material through fundoplication that allograft function is improved both through direct airway damage and through indirect augmented immunologic response to foreign antigen. Patients with more advanced stages of BOS did not have as reliable an improvement in terms of function. The pathologic changes of peribronchial fibrosis present in advanced stages of obliterative bronchiolitis are likely irreversible. However, the very consistent improvement in $\mathrm{FEV}_{1}$ after fundoplication must be compared with other treatment mo- dalities, usually involving augmented or altered immunosuppression, which has had limited success. ${ }^{9-18}$ Success in these series is defined by stabilization of the patient's $\mathrm{FEV}_{1}$ and rarely is associated with substantial improvements in spirometric function. Laproscopic Nissen fundoplication was well tolerated in this patient cohort without mortality and tolerable morbidity. From this data, we recommend in patients with declining $\mathrm{FEV}_{1}$ values that the patient be evaluated for the presence of GERD when consideration is being given to fundoplication. Because many of the patients might not have classic symptoms of GERD, a routine assessment with a pH probe probably is indicated.

A significant limitation of this study and all other studies using BOS criteria for defining chronic rejection is that decreasing $\mathrm{FEV}_{1}$ values in the absence of other treatable causes is a surrogate marker for obliterative bronchiolitis. The sensitivity for obliterative bronchiolitis, the pathologic correlative for chronic rejection, might be as low as 50\% on the basis of autopsy evaluation. Although the improvement in spirometry in those patients with GERD after lung transplantation who underwent fundoplication might not represent changes in immunologic or pathologic events, there was a substantial survival advantage in these patients. Although the cohort who underwent fundoplication was not randomly selected, patients selected for fundoplication, particularly early in our experience, represented a group of patients who already had demonstrated decreases in allograft function. Specifically, these patients were more likely to have BOS and had more frequent and higher grades of acute rejection. Despite these poor prognostic factors, these patients had improved survival and achieved survivals paralleling those for heart and kidney allografts.

The retrospective nature and patient selection bias are limitations of this study. Although the lung transplant recipients who had GERD and underwent fundoplication had decreasing spirometry results and impaired lung allograft function, they were believed to be acceptable candidates for surgical fundoplication. With the initial results demonstrating limited morbidity and no mortality and with demonstrable improvements in lung allograft function, fundoplication has been applied earlier and to less healthy patients. Although fundoplication was successful in improving lung allograft function, medical therapy with either proton pump inhibitors or histamine receptor antagonists did not appear to be as efficacious. Comparisons between those patients managed with fundoplication compared with those managed medically are not comparable in this series because of differences in comorbid variables. However, survival in the fundoplication cohort was better than that for the overall cohort, despite having more rejection episodes, more severe rejection episodes, and a higher incidence of BOS. Patients treated with a fundoplication, particularly early in this experience, had to survive the initial posttransplant period to 

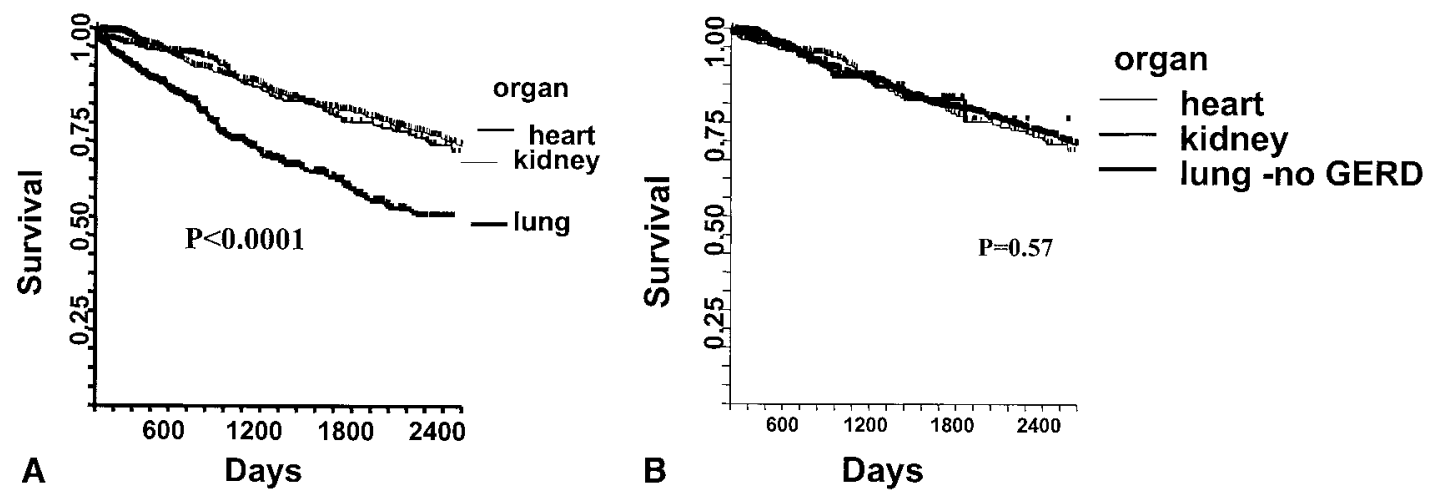

Figure 4. Kaplan-Meier actuarial allograft survival curves: allograft survival of at least 6 months to adjust for differences between organs on the basis of early technical variables. A, Overall allograft survival (6-month survivors) for patients undergoing kidney, heart, and lung transplantation, documenting a worse outcome in lung transplant recipients. B, Overall allograft survival in lung transplant recipients who did not have reflux or whose reflux was corrected by means of fundoplication compared with recipients of kidney or heart transplants. Allograft survival among the 3 groups was almost identical.

be able to receive this therapy. When these 2 groups were compared after eliminating the initial 6 months, thus removing the early mortality in the overall cohort, the difference in survival remained significant. This has led to a number of questions, including the following. When is the optimal time to proceed with fundoplication in patients after lung transplantation in documented GERD? Because of the impaired mucociliary function and abnormal cough reflex, what should the amount of acid contact time be to indicate candidacy for fundoplication?

Although this study cannot conclusively demonstrate a linkage between obliterative bronchiolitis or BOS and GERD, it does demonstrate that significant improvement in lung allograft function can be achieved through the use of fundoplication. Patients with declining $\mathrm{FEV}_{1}$ values, in addition to evaluation for acute rejection, airway complication, and infection, should also undergo evaluation for GERD. GERD appears to represent another category of disease processes affecting lung allografts that might be treatable and reversible. Whether prevention of reflux disease and other nonalloimmune injury can abrogate or prevent alloimmunologic injury should be a focus of future studies.

\section{References}

1. UNOS. US Scientific Registry of Transplant Recipients and The Organ Procurement and Transplantation Network: 1999 annual report-transplant data 1989-1998. Department of Health and Human Services. 1999.

2. Hosenpud J, Bennett L, Keck B, et al. The registry of the International Society for Heart and Lung Transplantation: Fifteenth official report. J Heart Lung Transplant. 1998;17:656-68.

3. Estenne M, Maurer J, Boehler A, et al. Bronchiolitis obliterans syndrome 2001: an update of the diagnostic criteria. J Heart Lung Transplant. 2002;21:297-310.
4. Cooper J, Billingham M, Eagan T, et al. A working formulation for the standardization of nomenclature and for clinical staging of chronic dysfunction in lung allografts. J Heart Lung Transplant. 1993;12: 713-6.

5. Kroshus TJ, Kshettry V, Savik K, et al. Risk factors for the development of bronchiolitis obliterans syndrome after lung transplantation. J Thorac Cardiovasc Surg. 1997;114:195-202.

6. Heng D, Sharples LD, McNeil K, et al. Bronchiolitis obliterans syndrome: incidence, natural history, prognosis, and risk factors. J Heart Lung Transplant. 1997;17:1255-63.

7. Sharples LD, McNeil K, Stewart S, Wallwork J. Risk factors for bronchiolitis obliterans: a systematic review of recent publications. J Heart Lung Transplant. 2002;21:271-81.

8. Boehler A, Estenne M. Obliterative bronchiolitis after lung transplantation. Curr Opin Pulm Med. 2000;6:133-9.

9. Snell G, Esmore D, Williams T. Cytolytic therapy for the bronchiolitis obliterans syndrome complicating lung transplantation. Chest. 1996; 109:874-8.

10. Kesten S, Rajagopalan N, Maurer J. Cytolytic therapy for the treatment of bronchiolitis obliterans syndrome following lung transplantation. Transplantation. 1996;61:427-30.

11. Speich R, Boehler A, Russi E, Weder W. A case report of a doubleblind, randomized trial of inhaled steroids in a patient with lung transplant bronchiolitis obliterans. Respiration. 1997;64:375-80.

12. Iacono A, Keenan RJ, Duncan S, et al. Aerosolized cyclosporine in lung recipients with refractory chronic rejection. Am J Respir Crit Care Med. 1996;153:1451-5.

13. Dusmet M, Maurer J, Winston T, Kesten S. Methotrexate can halt the progression of bronchiolitis obliterans syndrome in lung transplant recipients. J Heart Lung Transplant. 1996;15:948-54.

14. Reichenspurner H, Meiser B, Kur F, et al. First experience with FK506 for treatment of chronic pulmonary rejection. Transplant Proc. 1995;27:2009.

15. Kesten S, Chaparro C, Scavuzzo M, Gutierrez C. Tacrolimus as rescue therapy for bronchiolitis obliterans syndrome. J Heart Lung Transplant. 1997;16:905-12.

16. Speich R, Boehler A, Thurnheer R, Weder W. Salvage therapy with mycophenolate mofetil for lung transplantation bronchiolitis obliterans: importance of dosage. Transplantation. 1997;64:533-5.

17. Whyte RI, Rossi SJ, Mulligan MS, et al. Mycophenolate mofetil for obliterative bronchiolitis syndrome after lung transplantation. Ann Thorac Surg. 1997;64:945-8.

18. Diamond DA, Michalski JM, Lynch JP, Trulock EP 3rd. Efficacy of total lymphoid irradiation for chronic allograft rejection following 
bilateral lung transplantation. Int J Radiat Oncol Biol Phys. 1998;41: 795-800.

19. McKane BW, Trulock EP, Patterson GA, Mohanakumar T. Lung transplantation and bronchiolitis obliterans: an evolution in understanding. Immunol Res. 2001;24:177-90.

20. Lau CL, Palmer SM, Posther KE, et al. Influence of panel-reactive antibodies on posttransplant outcomes in lung transplant recipients. Ann Thorac Surg. 2000;69:1520-4.

21. Jaramillo A, Naziruddin B, Zhang L, et al. Activation of human airway epithelial cells by non-HLA antibodies developed after lung transplantation: a potential etiological factor for bronchiolitis obliterans syndrome. Transplantation. 2001;71:966-76.

22. Sundaresan S, Mohanakumar T, Smith MA, et al. HLA-A locus mismatches and development of antibodies to HLA after lung transplantation correlate with the development of bronchiolitis obliterans syndrome. Transplantation. 1998;65:648-53.

23. Palmer SM, Miralles AP, Howell DN, et al. Gastroesophageal reflux as a reversible cause of allograft dysfunction after lung transplantation. Chest. 2000;118:1214-7.

24. Rinaldi M, Martinelli L, Volpato G, et al. Gastro-esophageal reflux as cause of obliterative bronchiolitis: a case report. Transplant Proc. 1995;27:2006-7.

25. Tobin RW, Pope CE 2nd, Pellegrini CA, et al. Increased prevalence of gastroesophageal reflux in patients with idiopathic pulmonary fibrosis. Am J Respir Crit Care Med. 1998;158:1804-8.

26. Perrin-Fayolle M. Gastroesophageal reflux and chronic respiratory disease in adults. Influence and results of surgical therapy. Clin Rev Allergy. 1990;8:457-69.

27. Ledson MJ, Tran J, Walshaw MJ. Prevalence and mechanisms of gastro-oesophageal reflux in adult cystic fibrosis patients. $J R S o c$ Med. 1998;91:7-9.

28. Field SK, Sutherland LR. Does medical antireflux therapy improve asthma in asthmatics with gastroesophageal reflux?: a critical review of the literature. Chest. 1998;114:275-83.

29. Compte L, Garrigues V, Perpina M, Ponce J. Prevalence of gastroesophageal reflux in asthma. J Asthma. 2000;37:175-82.

30. Stringer DA, Sprigg A, Juodis E, et al. The association of cystic fibrosis, gastroesophageal reflux, and reduced pulmonary function. Can Assoc Radiol J. 1988;39:100-2.

31. Suen HC, Hendrix H, Patterson GA. Physiologic consequences of pneumonectomy. Consequences on the esophageal function. Chest Surg Clin North Am. 1999;9:475-83.

32. Sadoun D, Valeyre D, Cargill J, et al. Bronchiolite obliterante avec pneumonie en voie d'organisation en appearance cryptogenetique. Presse Med. 1988;17:2383-5.

33. Higgenbottam T, Jackson M, Woolman P. The cough response to ultrasonically nebulized distilled water in heart-lung transplantation patients. Am Rev Respir Dis. 1989;140:152-7.

34. Rivero DH, Lorenzi-Filho G, Pazetti R, et al. Effects of bronchial transection and reanastomosis on mucociliary system. Chest. 2001; 119:1510-5.

35. Tomkiewicz RP, App EM, Shennib H, et al. Airway mucus and epithelial function in a canine model of single lung autotransplantation. Chest. 1995; 107:261-5.

36. Veale D, Glasper PN, Gascoigne A, et al. Ciliary beat frequency in transplanted lungs. Thorax. 1993;48:629-31.

37. Herve P, Silbert D, Cerrina J, et al. Impairment of bronchial mucociliary clearance in long-term survivors of heart/lung and double-lung transplantation. The Paris-Sud Lung Transplant Group. Chest. 1993; 103:59-63.

38. Richter JE. Ambulatory esophageal pH monitoring. Am J Med. 1997; 103:130S-4S.

39. Mays EE, Dubois JJ, Hamilton GB. Pulmonary fibrosis associated with tracheobronchial aspiration. A study of the frequency of hiatal hernia and gastroesophageal reflux in interstitial pulmonary fibrosis of obscure etiology. Chest. 1976;69:512-5.

\section{Discussion}

Dr G. Alexander Patterson (St Louis, Mo). This was an excellent presentation. I enjoyed it for 2 reasons. First because it was delivered by Duane Davis, who, when he returned to Duke after a fellowship in our program some years ago, resuscitated a moribund transplant program. As you can see from their 400 cases in a decade, Duke has acquired a big programmatic experience. Dr Davis has transformed that program from virtually nothing to one of the most active and productive lung transplant programs in the world. He is recognized increasingly as an important contributor in transplantation.

I also think the message is important. Bronchiolitis obliterans is the biggest obstacle to long-term success in lung transplantation. I also think that virtually everyone who leaves the operating room after a bilateral lung transplantation has significant vagal dysfunction. I suspect a good number of those patients have been completely vagotomized by that operation, and I do not doubt that this predisposes patients to increased reflux, particularly in a group of patients who might be predisposed to reflux because of their underlying lung disease.

A previous speaker showed us a beautiful slide of bronchiolitis obliterans, the pathologic lesion. He also described the clinical scoring system to describe BOS. I cannot believe that the pathologic lesion can be reversed with an antireflux operation or a fundoplication. We should also remember that bronchiolitis obliterans, the pathologic lesion, was described long before anybody underwent lung transplantation. In fact, in the transplantation context bronchiolitis obliterans was first described in patients undergoing bone marrow transplantation.

Therefore it is not entirely clear to me what is being reversed. I do not doubt that there are patients whose lung function is improved, and therefore their BOS score is improved, but I do not believe that their bronchiolitis obliterans is being corrected with antireflux surgery.

I do have a number of questions. Duane, I am not sure what evidence we have, apart from clinical conviction, that these patients are indeed vagotomized. Do you have any data about acid secretion or response to sham feeding? And if these patients do have a vagal injury, do you think it is permanent or do you think it somehow resolves?

We have seen many transplant recipients with large distended stomachs, air-fluid levels, retained food in the stomach, and delayed gastric emptying. Have any of your patients undergone a drainage procedure in addition to their fundoplication?

I also would like your thoughts about what pathologic lesion is being improved or reversed to demonstrate such an impressive improvement in $\mathrm{FEV}_{1}$ values and BOS scores.

Finally, I know that you have given some thought to subjecting this to a prospective trial. Do you really think that that is necessary given the compelling data that you have presented here today?

Dr Davis. Thank you very much for your kind comments, Dr Patterson. I am going to take the last one first.

The importance of doing a randomized trial is that I do not think we understand the interaction between obliterative bronchiolitis and BOS. A nice autopsy study was presented about 3 weeks ago demonstrating that essentially $50 \%$ of the patients who died of obliterative bronchiolitis actually died of other causes. Therefore the use of BOS as a surrogate marker for obliterative bronchiolitis or the pathologic lesion is difficult at best. However, we need 
something that is sensitive enough to pick up those patients with obliterative bronchiolitis.

We need to understand better the pathophysiologic or immunologic injuries that are involved in lung allograft dysfunction. The context of when alloimmune presentation occurs is being very important in terms of the type of immunologic response. Why shouldn't the lung be more like the liver and be more-I will not say tolerogenic_-but more anergic in its response? Yet we seem to have the clinical problem of having increased alloimmune responses. Therefore could it be that what we do with reflux injury is that we actually present alloantigens at a time in which there is an inflammatory response present, so that you increase the number of peptides that can actually be presented to immune-responding cells in the context of increased costimulatory singling et cetera? The idea would be to put patients into a randomized trial. We have demonstrated that we can salvage most patients who meet BOS 1 criteria and bring them back out of BOS, so that we have a safety net. However, the idea of actually studying it in the context of differences in the immunologic response, whether of the direct pathway or the indirect pathway, would be very interesting and potentially very important.

With regard to the proof of the vagal injury, we have not done that in our transplant recipients. It is actually a very useful and important question. Dr Steve Eubanks, one of our collaborators, has a wonderful dog model for reflux disease, and his group is actually studying what we are seeing clinically in that model. Whether the various immunosuppressants also have a strong component can be examined in our non-lung transplant population, to just see what the effect is in the patients undergoing kidney and heart transplantation.

In terms of drainage procedures, we have done a number of drainage procedures, particularly with procedures early after transplant. Those patients tended to have a longer length of stay and a little more complicated course. What we have convinced our laparoscopic surgeons to do now is to place a formal gastrostomy tube and jejunostomy tube in addition to the fundoplication and to use the gastrostomy tube to decompress the stomach for a short period of time. Most patients by the 3- to 6-month period actually recover a substantial amount of their gastrointestinal function.

In terms of what the pathologic lesion is, I do not think that it is the fibrotic lesion or the fibroproliferative lesion that was demonstrated in the previous talk. That, I think, is gone, water under the bridge. We cannot chase that. I think that is the effect that we see when we try to do these operations for the patients with BOS 3. We are not going to reverse that process. We might be able to stabilize it and prevent additional injury, and we certainly have done that, staging patients who are going on to have retransplantations for obliterative bronchiolitis, but if we actually are getting to that point, we have nothing really to offer in terms of really reversing the process.

Dr Hiran C. Fernando (Pittsburgh, $\mathrm{Pa}$ ). Dr Davis, I enjoyed your presentation. I have a couple of questions.

Do you have any idea how successful the laparoscopic fundoplication was in terms of 24-hour $\mathrm{pH}$, proton pump inhibitor use, or symptom severity scores and whether the success of the operation correlated with your outcome?
In your patient group, did you have any patients with scleroderma who needed antireflux surgery, and if so, what type of operation was performed?

Dr Davis. With regard to the laparoscopic procedure, we have not routinely studied this, but all the patients who have had a postoperative 24-hour $\mathrm{pH}$ study done have had essentially no reflux, which I think is fairly important in terms of a randomized trial. There are 2 questions: What can you do clinically, and, from a mechanism standpoint, what is the role of reflux? Therefore it is going to be important to study prospectively what happens after fundoplication. That gets into the question of what is enough reflux to put somebody through an operation. The lungs are denervated. The mucociliary function is very poor. Anything that gets into the lungs is going to sit there, and it is going to cause more injury than in anyone with a normal lung, and in the face of having alloimmune responses instead of syngeneic responses, you are going to have more of an injury response.

With regard to the scleroderma, we do not categorically rule it out, but if the esophageal function is very depressed, we do not take those patients on primarily for this problem.

Dr Malcolm M. DeCamp (Cleveland, Ohio). Along the same lines, in trying to bring this issue of reflux into the pretransplant evaluation, is there a profile of a patient with reflux who you would not transplant or who you would push toward fundoplication before transplantation to try to avoid dealing with the problem during the early posttransplant period? At the Brigham \& Women's Hospital, we found that we were recovering oral flora and gastrointestinal flora 3 times more commonly in protected brush specimens in the patients undergoing transplantation, even without a prior history of reflux disease. Therefore I think you have helped define an important point; we definitely disturb esophageal and gastric function after this operation. Perhaps we ought to pay more attention to it in our pretransplant evaluation.

Dr Davis. We prospectively study everyone at this point because of some of those data. We do not turn down patients on the basis of just the presence of reflux if they have normal esophageal function. What we have done more and more is subject those patients to very early fundoplication, and in fact, if they in the immediate posttransplant period have what appears to be an aspiration episode and get reintubated, we actually put them through a fundoplication before we get them extubated. But the presence of it beforehand does not exclude candidacy for lung transplantation. It just raises awareness.

Dr Ross M. Bremner (Los Angeles, Calif). Do you have any manometric data on these patients, both preoperatively and postoperatively? That might help us elucidate whether this is an esophageal body problem, a sphincter problem, or, in fact, a gastric emptying problem. The latter might be related, in part, to possible vagal damage at the time of transplantation, as inferred by Dr Patterson.

Second, have you had an opportunity to study any of these patients with a dual-lumen $\mathrm{pH}$ probe or a probe that is placed at the level of the laryngeal aditus to see if in fact some of this reflux is reaching that area?

Dr Davis. With regard to manometry, the vast majority of the patients have normal esophageal function and normal lower esophageal sphincter function. Therefore in some ways we are excluding the patients who really have tremendous esophageal body problems, and in fact, some of the medically treated patients probably 
were turned down for fundoplication early on because of concerns about drainage, movement, the esophageal function.

As to your second question, the fact is, what you see distally is what you see proximally, more so than in the pretransplantation patient. There is a bit of a degree of absolute change, so that if you are seeing a $10 \%$ acid contact time in the distal probe, it is usually about $7 \%$ in the proximal probe. We have never repeated the experience where you put the tracheal $\mathrm{pH}$ probes in. We just have not been able to get much buy in on that.

Dr. Walter Klepetko (Vienna, Austria). I think this is a very important contribution.

One would assume that patients with significant reflux also have a variety of other motility disorders, and as we all know, those patients tend to have difficulties in achieving constant immunosuppressive levels. Did you have the chance to compare those values before and after fundoplication? Although I would not expect that the fundoplication itself has any effect on that, it would be of interest to hear whether those patients in particular had a highly variable level of immunosuppression.

Dr. Davis. In terms of what is happening in this patient cohort, there has been no difference in the amount of immunosuppression. As a matter of fact, most of those patients, particularly the ones that hit BOS $1, \operatorname{BOS} 2$, or BOS 3 categories, have already gone through a whole variety of changes in the immunosuppression and augmentation of the immunosuppression. To get around the variability with absorption from the gastrointestinal system, we have used sublingual tacrolimus, and it is absorbed wonderfully. In fact, we stay with sublingual tacrolimus as long as there is an issue with the gut function, and that way we are able to achieve therapeutic levels consistently. 